\title{
Coronal emission from the shocked circumstellar ring of SN 1987A
}

\author{
P. Gröningsson ${ }^{1}$, C. Fransson ${ }^{1}$, P. Lundqvist ${ }^{1}$, T. Nymark ${ }^{1}$, N. Lundqvist $^{1}$, R. Chevalier ${ }^{2}$, \\ B. Leibundgut ${ }^{3}$, and J. Spyromilio ${ }^{3}$
}

\footnotetext{
1 Department of Astronomy, Stockholm University, AlbaNova University Center, 10691 Stockholm, Sweden

2 Department of Astronomy, University of Virginia, PO Box 3818, Charlottesville, VA 22903, USA

3 European Southern Observatory, Karl-Schwarzschild-Strasse 2, 85748 Garching, Germany

e-mail: per@astro.su.se
}

Received 30 March 2006 / Accepted 9 June 2006

ABSTRACT

\begin{abstract}
High resolution spectra with UVES/VLT of SN 1987A from December 2000 until November 2005 show a number of high ionization lines from gas with velocities of $\sim \pm 350 \mathrm{~km} \mathrm{~s}^{-1}$, emerging from the shocked gas formed by the ejecta-ring collision. These include coronal lines from $[\mathrm{Fe} \mathrm{X}]$, [Fe XI] and [Fe XIV] which have increased by a factor of $\sim 20$ during the observed period. The evolution of the lines is similar to that of the soft X-rays, indicating that they arise in the same component. The line ratios are consistent with those expected from radiative shocks with velocity $310-390 \mathrm{~km} \mathrm{~s}^{-1}$, corresponding to a shock temperature of $(1.6-2.5) \times 10^{6} \mathrm{~K}$. A fraction of the coronal emission may, however, originate in higher velocity adiabatic shocks.
\end{abstract}

Key words. supernovae: individual: SN 1987A - circumstellar matter - shock waves

\section{Introduction}

The collision of the ejecta of SN 1987A with the circumstellar ring has been observed in many wavelength ranges (e.g., McCray 2005 for a review). In the UV/optical range this has been monitored with HST showing an increasing number of hot spots around the ring (Michael et al. 2000, 2002; Pun et al. 2002). Most likely, these hot spots are caused by the impact of the blast wave on protrusions from the inner circumstellar ring. As time progresses an increasing number of these are expected to appear, and finally the whole ring will be immersed in the collision.

The spectroscopic HST observations have identified three different velocity components (Pun et al. 2002). One narrow, nearly Gaussian, with $F W H M \sim 10 \mathrm{~km} \mathrm{~s}^{-1}$, coming from the unshocked ring gas. Secondly, there is an intermediate velocity component arising from shocked cooling gas, with a clearly non-Gaussian form, extending to $\sim \pm 300 \mathrm{~km} \mathrm{~s}^{-1}$. Finally there is a very broad component extending to $\sim 15000 \mathrm{~km} \mathrm{~s}^{-1}$, probably coming from the reverse shock, resulting from the interaction with the surrounding medium (Michael et al. 1998; Smith et al. 2005). We will refer to these as the narrow, intermediate and high velocity components, respectively.

The collision is also seen in the radio as a rising non-thermal synchrotron flux (Gaensler et al. 1997; Manchester et al. 2002, 2005). The light curves in this range show a roughly linear increase with time. Further, Bouchet et al. (2006) find from ground based and Spitzer mid-IR observations evidence for hot dust as well as highly ionized line emission.

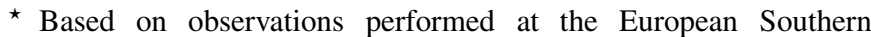
Observatory, Paranal, Chile.
In X-rays ROSAT and Chandra have observed SN 1987A at several epochs, and also in this energy range a steadily rising flux is seen (Park et al. 2004, 2005a). While the hard X-rays correlate well with the radio flux, the rise time in the soft X-rays is considerably shorter. Of special interest are the high resolution spectral observations (Zhekov et al. 2005, 2006), which show a number of lines from $\mathrm{H}$ and $\mathrm{He}$ like ions of $\mathrm{N}, \mathrm{O}, \mathrm{Ne}, \mathrm{Si}, \mathrm{Mg}$ and $\mathrm{S}$, as well as Fe XVII. Zhekov et al. (2005) argue that these lines arise in the radiative shocks propagating into the protrusions, as well as the reflected shock propagating back from these. In this paper we discuss optical observations complementary to these X-ray observations of highly ionized gas.

In Gröningsson et al. (2006) we report on spectral shapes and fluxes of optical lines created by the ejecta/ring interaction. As we use high-resolution echelle spectroscopy, we can easily disentangle the different velocity components from each other. Here we concentrate on the evolution of intermediate velocity high-ionization lines, such as [Fe X] 16374.5 , [Fe XI] $\lambda 7891.8$ and [Fe XIV] 25302.9, as they are efficient probes of the shocked gas.

In our analysis we use a recession velocity of $281 \mathrm{~km} \mathrm{~s}^{-1}$ (the bulk motion of the northern side of the inner circumstellar ring (Gröningsson et al. 2006)) and a reddening of $E_{B-V}=0.16$ (Fitzpatrick \& Walborn 1990) with $E_{B-V}=0.06$ from the Milky way (e.g., Staveley-Smith et al. 2003) and $E_{B-V}=0.10$ from the LMC. The reddening law was taken from Cardelli et al. (1989) using $R_{V}=3.1$. The differences between the LMC extinction law and the Galactic law are negligible in the optical at low color excess (Fitzpatrick 1999) and have therefore been ignored. In Sects. 2 and 3 we describe the observations and observational results, respectively. In Sect. 4 we discuss modeling 
Table 1. Log of UVES observations.

\begin{tabular}{|c|c|c|c|c|c|c|c|c|}
\hline$\overline{\text { Epoch }}$ & Date & $\begin{array}{l}\text { Days after } \\
\text { explosion }\end{array}$ & Grating & $\lambda \underset{\AA}{\lambda \text { range }}$ & $\begin{array}{l}\text { Slit width } \\
\text { arcsec }\end{array}$ & $\begin{array}{c}\text { Resolution } \\
\lambda / \Delta \lambda\end{array}$ & $\begin{array}{c}\text { Exposure } \\
\text { s }\end{array}$ & $\begin{array}{l}\text { Seeing } \\
\text { arcsec }\end{array}$ \\
\hline 1 & 1999 Oct. 16 & 4618 & DIC1 346+580 & $\begin{array}{l}3100-3900 \\
4800-6800\end{array}$ & 1.0 & 40000 & 1200 & 1.0 \\
\hline \multirow[t]{2}{*}{2} & \multirow[t]{2}{*}{2000 Dec. $9-14$} & 5039-5043 & DIC1 $346+580$ & $\begin{array}{l}3100-3900 \\
4800-6800\end{array}$ & 0.8 & 50000 & 10200 & $0.4-0.8$ \\
\hline & & $5038-5039$ & DIC2 390+860 & $\begin{array}{c}3300-4400 \\
6700-10000\end{array}$ & & & 9360 & 0.4 \\
\hline \multirow[t]{2}{*}{3} & \multirow[t]{2}{*}{2002 Oct. 4-7 } & 5704-5705 & DIC1 $346+580$ & $3100-3900$ & 0.8 & 50000 & 10200 & $0.7-1.0$ \\
\hline & & $5702-5703$ & DIC2 $437+860$ & $\begin{array}{c}4800-0800 \\
3800-4900 \\
6700-10000\end{array}$ & & & 9360 & $0.4-1.1$ \\
\hline \multirow[t]{2}{*}{4} & \multirow{2}{*}{$\begin{array}{l}2005 \text { Mar. } 21 \\
\text { Apr. } 8-12\end{array}$} & $6601-6623$ & & $\begin{array}{l}3100-3900 \\
4800-6800\end{array}$ & 0.8 & 50000 & 9200 & $0.6-0.9$ \\
\hline & & $6621-6623$ & DIC2 $437+860$ & $\begin{array}{c}3800-4900 \\
6700-10000\end{array}$ & & & 4600 & 0.5 \\
\hline \multirow[t]{2}{*}{5} & \multirow[t]{2}{*}{$\begin{array}{l}2005 \text { Oct. } 20 \\
\text { Nov. } 1-12\end{array}$} & 6826 & DIC1 $346+580$ & $\begin{array}{l}3100-3900 \\
4800-6800\end{array}$ & 0.8 & 50000 & 2300 & 0.9 \\
\hline & & $6814-6837$ & DIC2 $437+860$ & $\begin{array}{c}3800-4900 \\
6700-10000\end{array}$ & & & 9200 & $0.5-1.0$ \\
\hline
\end{tabular}

of the lines, followed by a general discussion in Sect. 5 and conclusions in Sect. 6.

\section{Observations}

SN 1987A was observed in service mode with the Ultraviolet and Visual Echelle Spectrograph (UVES) at ESO/VLT at Paranal, Chile. UVES is a cross-dispersed echelle spectrograph covering (with two different dichroic settings) the spectral range 23100-10000 $\AA$ (Dekker et al. 2000). The light beam is split up in two separate arms. The arm covering the blue part of the spectrum ( 3100-4900 $\AA$ ) has a single CCD detector with a spatial resolution of $0.246 /$ pix. The other arm covering the red part ( 4800-10000 $\AA$ ) is equipped with two CCDs having the somewhat higher spatial resolution of 0 .' $182 /$ pix. The slit width used resulted in a resolving power of $\sim 50000$ corresponding to a spectral resolution of $\sim 6 \mathrm{~km} \mathrm{~s}^{-1}$.

Spectra of the ring and ejecta of SN 1987A were obtained on October 16, 1999, December 9-14, 2000, October 4-7, 2002, March 21-April 12, 2005 and October 20-November 12, 2005, in the following referred to as epochs $1-5$, respectively. In all cases, except for epoch 1 , the position angle was $\mathrm{PA}=30^{\circ}$. For epoch 1 it was $\mathrm{PA}=20^{\circ}$. The log of the observations is given in Table 1. The observations and data reduction are discussed in detail in Gröningsson et al. (2006). As can be seen from Table 1, the exposure of the first epoch was very short and the $\mathrm{S} / \mathrm{N}$ low. This made it impossible to obtain meaningful fluxes for the coronal lines for this epoch, and therefore we do not discuss it further in this paper.

Because the spatial resolution of these ground-based observations is limited compared to the dimensions of the ring, we cannot distinguish between different hot spots located on the same side of the ring. To identify how many hot spots were covered by the slit we retrieved and studied HST images taken with the WFPC2 and ACS instruments at roughly the same epochs. These show that only Spot 1 is responsible for the emission from the shocked ring at epoch 1 . At epoch 2 Spot 1 still dominates the shocked gas emission on the north side of the ring, but also two spots on the opposite side of the ring are now prominent. At epoch 3, three to four different hot spots contribute to the emission on the northern side and by the time of the last epochs the entire ring is lit up by the ejecta-ring collision. Because of the difference in velocity and to isolate a limited part of the ring we will in the following only discuss the spectrum from the north side of the ring, i.e., where Spot 1 first appeared. A comparison of the the kinematics and flux of the two sides will be given in Gröningsson et al. (2006).

To estimate the accuracy of the absolute flux level of the spectra we compared the individual sensitivity functions created from the reduced standard star spectra and their corresponding physical fluxes. The sensitivity curves differed typically by $\$ 10 \%$ from each other. These exposures of the standard stars used a wide slit $\left(10^{\prime \prime}\right)$ and hence no slit losses are likely to occur. Our science data exposures, on the other hand, used a relatively narrow slit and since SN 1987A is an extended source the slit losses could be considerable and depend on atmospheric conditions such as the seeing. To estimate how much the atmospheric conditions could influence our results we have compared line fluxes of the individual science spectra for the epochs which have more than one exposure. This comparison reveals that the line fluxes differ by $\lesssim 10 \%$ from one exposure to another. Finally, to estimate the total systematic error, we have compared our fluxes with HST spectra and photometry (see Gröningsson et al. 2006), and find that the accuracy of the VLT fluxes should in general be better than $\sim 30 \%$.

\section{Results}

In Gröningsson et al. (2006) we give a full list of the different lines present, both from the unshocked and the shocked ring. The former are dominated by thermal broadening with a FWHM of $\sim 10 \mathrm{~km} \mathrm{~s}^{-1}$. The lines from the shocked gas are on the other hand dominated by the macroscopic velocity and are highly asymmetrical, extending to $\sim 250-400 \mathrm{~km} \mathrm{~s}^{-1}$ (HWZI). The two components can therefore easily be well separated, and 

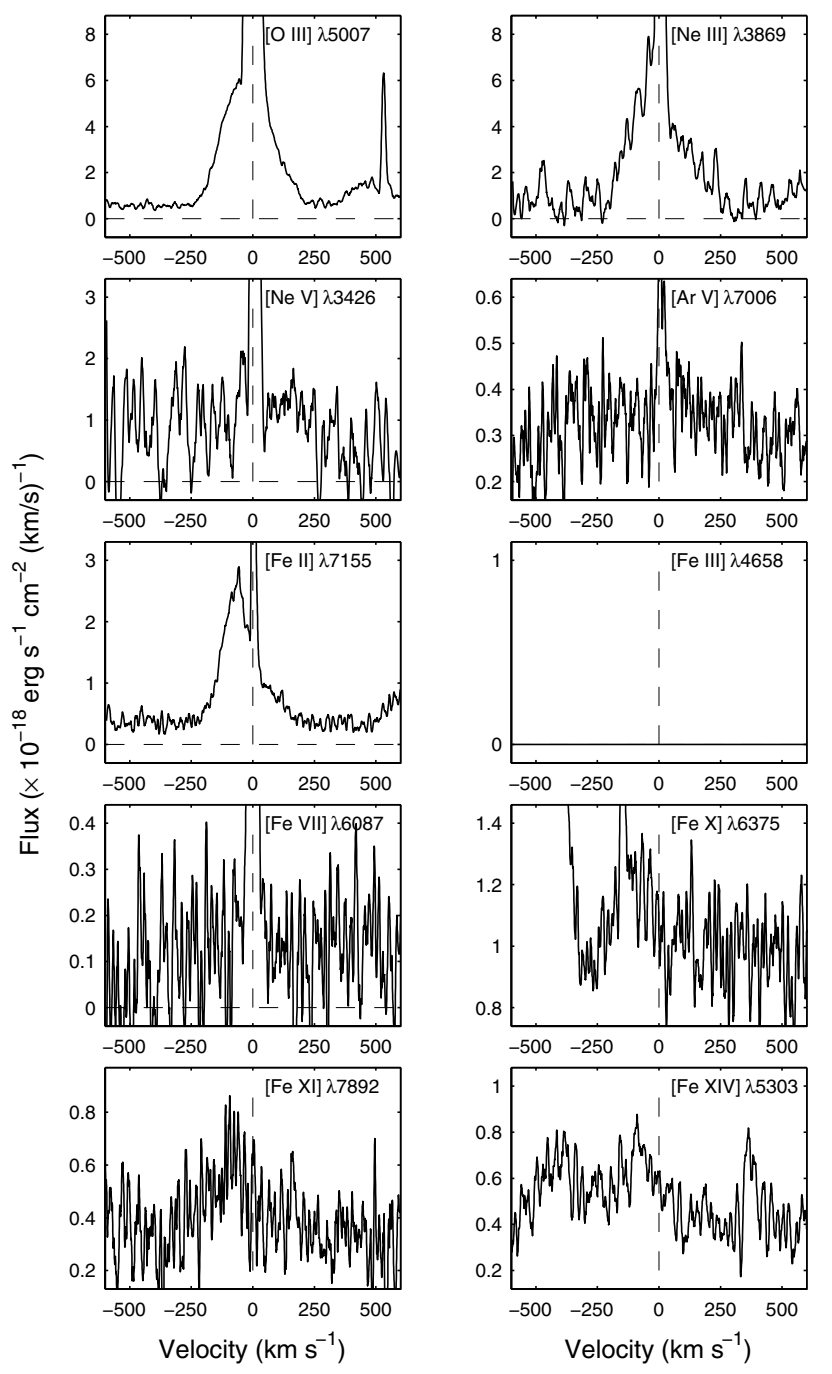

Fig. 1. Original line profiles from December 2000 (our epoch 2) of the most important high ionization lines from the north side of the shocked ring. The $[\mathrm{Fe} \mathrm{III}] \lambda 4658.1$ line was not covered by the observations at this epoch. Note the different flux scales. All velocities are with respect to the systemic velocity of the northern part of the ring, $281 \mathrm{~km} \mathrm{~s}^{-1}$.

a subtraction of the broader from the narrower component by interpolation of the intermediate components by regression splines is fairly straightforward. More problematic is the blending of different lines from the shocked component. In particular, the large number of Fe II lines from this component causes a considerable number of blendings.

The full spectrum between $3100-10000 \AA$ contains $\sim 170$ intermediate velocity lines. About one third of these are Fe II lines, but there are also strong lines from more highly ionized ions, like [O III] $\lambda \lambda 4958.9,5006.8,[\mathrm{Ne}$ III] $\lambda \lambda 3868.9,3967.5$, $[\mathrm{Ne} \mathrm{V}] \lambda \lambda 3345.3,3425.9$, and [Ar III] $\lambda 7135.8$. The most interesting result is, however, that we find a number of highly ionized coronal lines, which can only be seen thanks to the high $\mathrm{S} / \mathrm{N}$ and high spectral resolution of our UVES observations.

In Figs. 1-4 we show a compilation of the most important of these lines for the December 2000, October 2002, March/April 2005 and November 2005 epochs. These in-

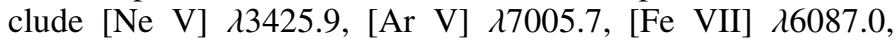
$[\mathrm{Fe} \mathrm{X}] \lambda 6374.5$, [Fe XI] $\lambda 7891.8$, and [Fe XIV] $\lambda$ 5302.9, which are all likely to originate from the cooling post-shock gas. In addition, we include for comparison the [O III] 15006.8 ,
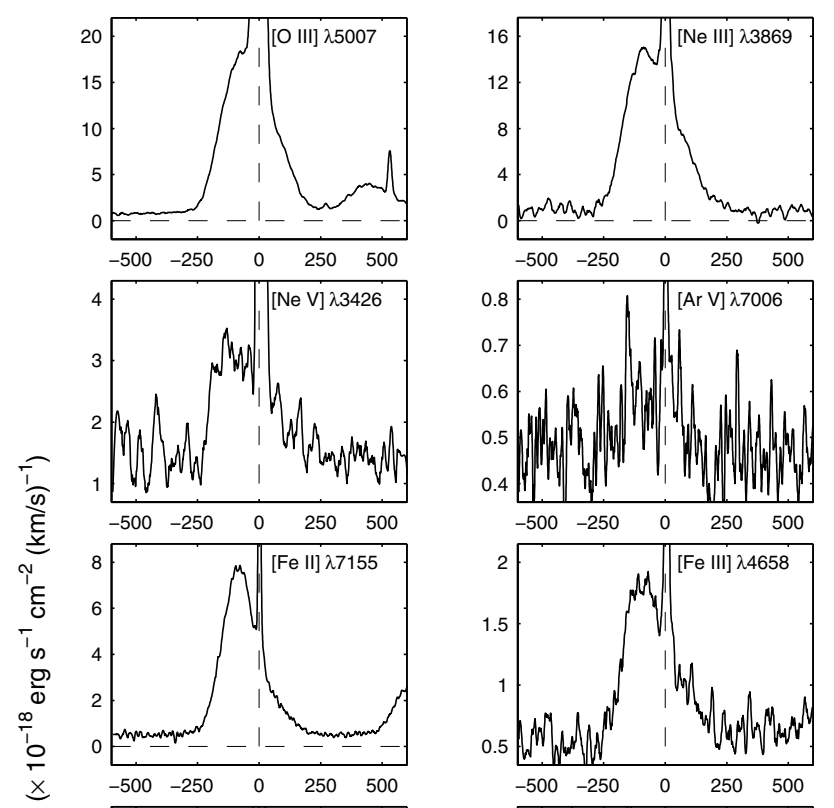

$\frac{x}{\underline{3}}$
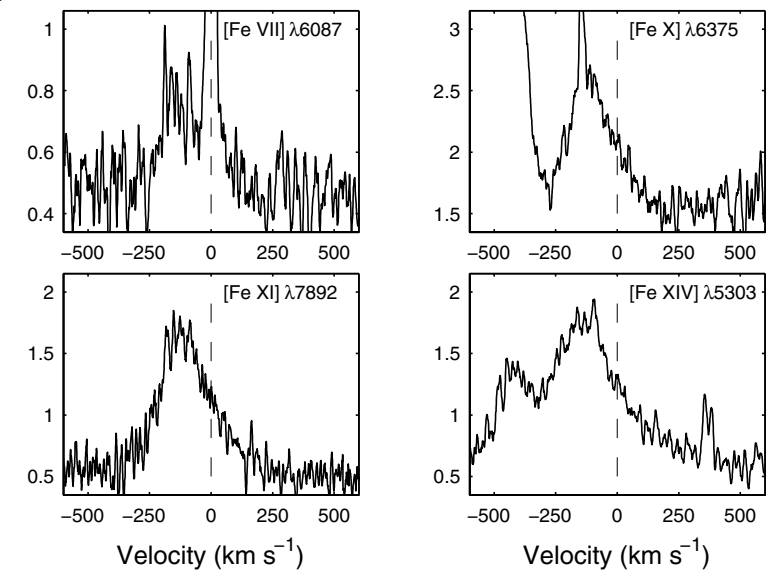

Fig. 2. Same as Fig. 1, but for October 2002.

$[\mathrm{Ne}$ III] $\lambda 3868.8,[\mathrm{Fe}$ II] $\lambda$ 77155.2, [Fe III] $\lambda 4658.0$ lines, which are, however, likely to come from considerably cooler, photoionized gas (Sect. 4). Because these are very useful as diagnostics of the shocked ring gas we concentrate in this paper on these lines, and refer to Gröningsson et al. (2006) for a more detailed analysis of the full spectrum.

As an important example we show in Fig. 5 the region of the [Fe XIV] $\lambda 5302.9$ line. It is here seen that the blue wing of the [Fe XIV] $\lambda 5302.9$ line is blended with the intermediate velocity [Fe II] $\lambda 5296.8$ line. To remove this we use the [Fe II] $\lambda 5527.6$ line as a template. The reason why we have chosen this line instead of the stronger [Fe II] $\lambda 7155.2$ line is the similar excitation potential to the [Fe II] $\lambda 5296.8$ line. The result of this subtraction can be seen in the lower panel of Fig. 5. In addition, we also see a narrow component of [Ca V] $\lambda 5309.1$ at $350 \mathrm{~km} \mathrm{~s}^{-1}$.

The $[\mathrm{Fe} \mathrm{X}] \lambda 6374.5$ line is close to the red wing of the very strong [O I] $\lambda 6363.8$ line. In addition, there is a narrow Si II $\lambda 6371.4$ line close to its peak. The [O I] $\lambda 6300.3$ line was used as a template to deblend [O I] $\lambda 6363.8$ from the $[\mathrm{Fe} \mathrm{X}] \lambda 6374.5$ line. The extension of the blue wing is therefore for this line uncertain.

In Fig. 6 we show the most important high ionization lines from the October 2002 observations after deblending. The other epochs have been deblended in the same manner. 

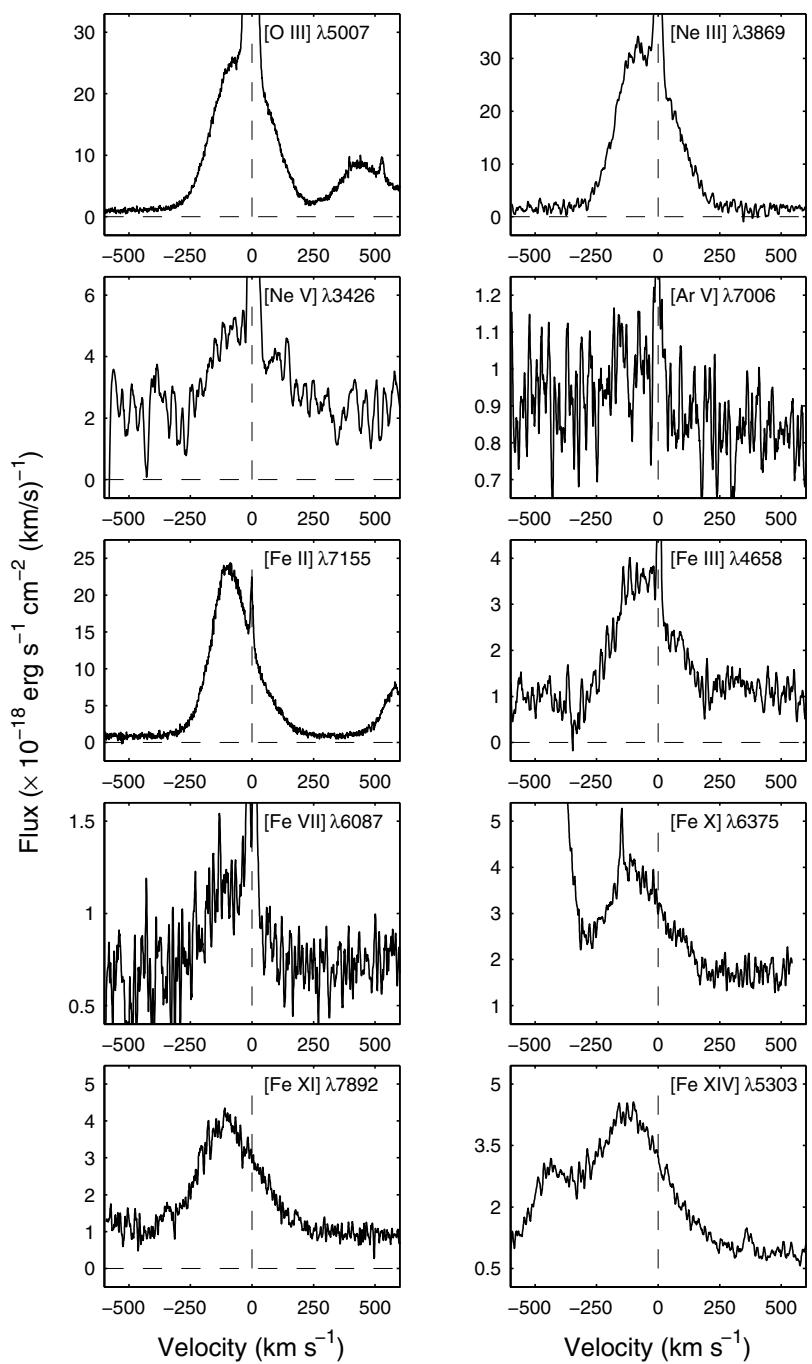

Fig. 3. Same as Fig. 1, but for March-April 2005.

We have also searched the spectra for [S XII] $\lambda 7611.0$, [Ar XIV] $\lambda 4412.2$ and [Ca XV] $\lambda \lambda 5446.4,5694.4$. The [S XII] line unfortunately falls on top of some very strong atmospheric bands. While in the data there is some evidence that a line may be present at the wavelength of the [S XII] line we cautiously attribute this to the effects of the atmosphere. We have also searched HST spectra in this spectral region. Because of the low dispersion, $4.92 \AA \mathrm{pixel}^{-1}$ or $194 \mathrm{~km} \mathrm{~s}^{-1}$ pixel $^{-1}$, and fairly low S/N, only a weak upper limit of $\sim 10^{-15} \mathrm{erg} \mathrm{s}^{-1}$ for the flux around our epoch 3 can be set.

In the region of the [Ar XIV] 14412.2 line there is a line redshifted from this wavelength by $\sim 200-300 \mathrm{~km} \mathrm{~s}^{-1}$ in our VLT spectra. This is, however, likely to be a blend of [Fe II] $\lambda \lambda 4413.8,4416.3$, which matches well with the velocity of the observed feature. Subtracting these lines, using [Fe II] $\lambda 7155.2$ as a template in the best $\mathrm{S} / \mathrm{N}$ spectrum of this region at epoch 5 , we estimate an upper limit of $\sim 15 \%$ of the [Fe XIV] flux at this epoch, i.e. $\$ 2.2 \times 10^{-16} \mathrm{erg} \mathrm{s}^{-1} \mathrm{~cm}^{-2}$.

We have also looked for the [Ca XV] 15694.4 line, but do not detect this to a flux limit of $\sim 15 \%$ of [Fe XIV] $\lambda 5302.9$ at epoch 4.

In Table 2 we give the fluxes of the intermediate velocity component for the highly ionized lines in the spectrum at the four well observed epochs. These fluxes are corrected for blending, as described above, but not for reddening. The estimated
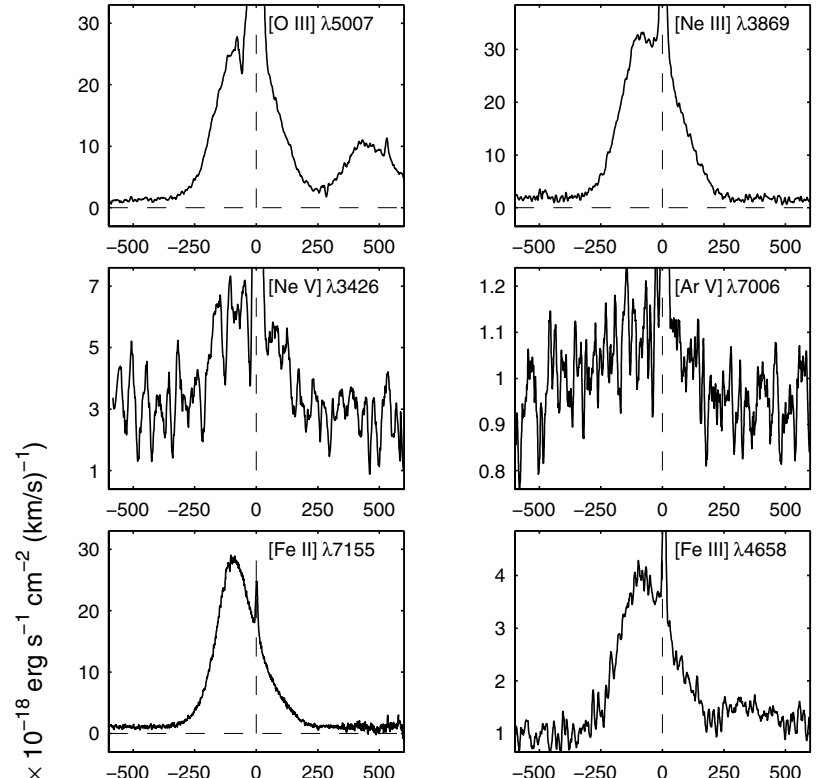

$\stackrel{x}{\frac{x}{4}}$
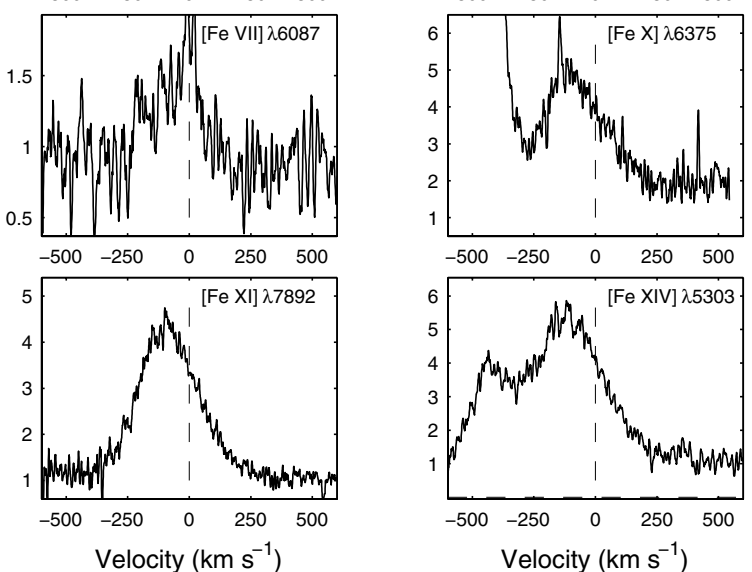

Fig. 4. Same as Fig. 1, but for November 2005.

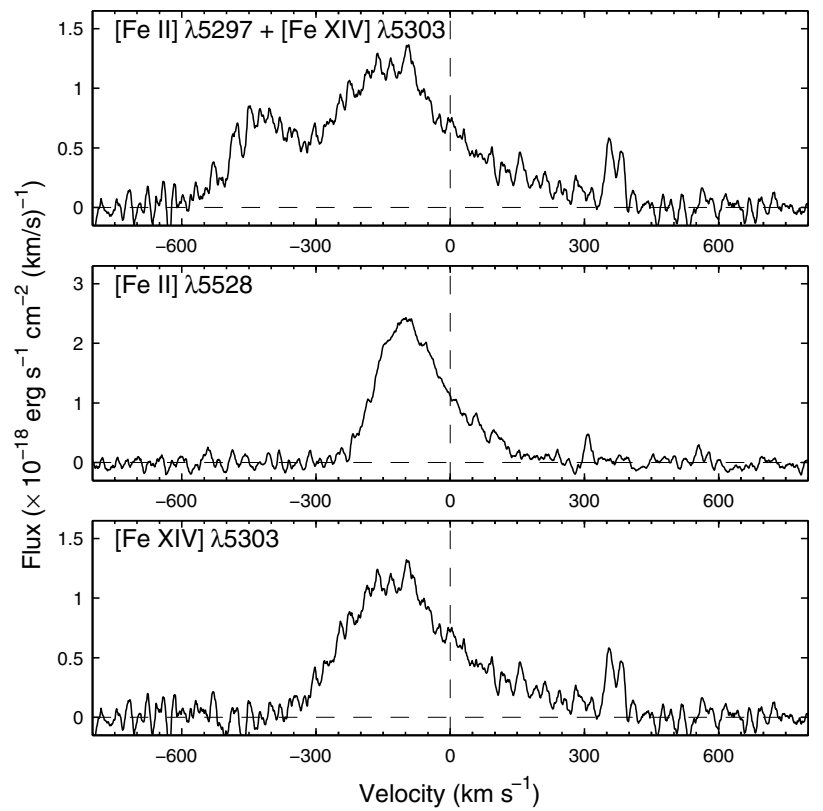

Fig. 5. Top panel: line profile of [Fe XIV] $\lambda 5302.9$, before deblending. Middle panel: template profile for [Fe II] $] 25296.8$, based on the [Fe II] $\lambda 5527.6$ line. Bottom panel: deblended profile. The narrow line at $\sim 350 \mathrm{~km} \mathrm{~s}^{-1}$ in the top and bottom panels is [Ca V] $\lambda 5309.1$. 

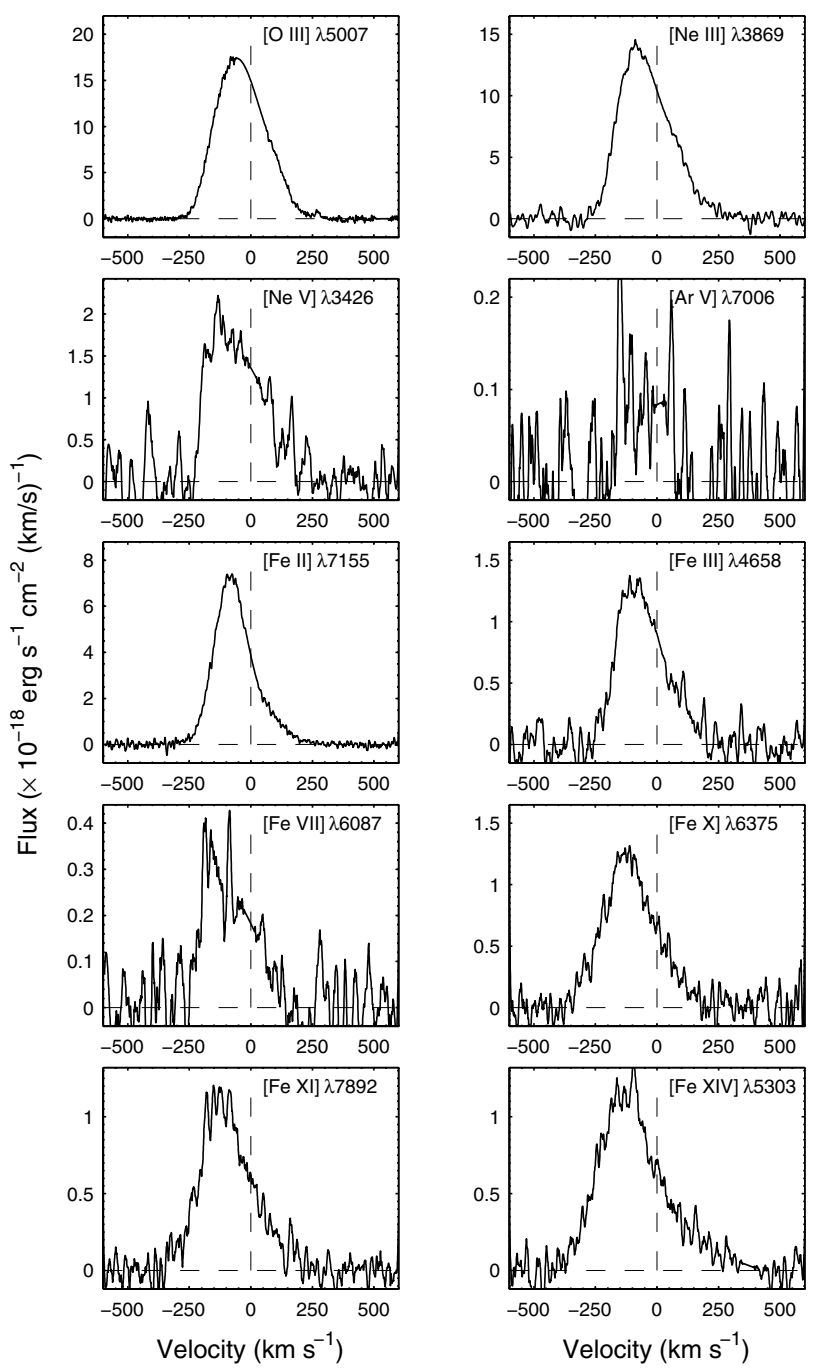

Fig. 6. Deblended line profiles from October 2002 of the high ionization lines in Fig. 2.

extinction correction factors are listed in Table 2. In addition, we detect the [Fe VI] $\lambda 5335.2$ line at epoch 4 with a flux of $(1.3 \pm 0.2) \times 10^{-16} \mathrm{erg} \mathrm{s}^{-1} \mathrm{~cm}^{-2}$.

As we see from Table 2 and Figs. 1-4, there is an increase in the fluxes of all lines between epochs 2 and 5. Over this period the $[\mathrm{Fe} \mathrm{X}]$, [Fe XI], and [Fe XIV] lines have increased by a factor 24-30. At epoch 2 some of the weaker lines are, however, close to the noise level, and only the fluxes at epoch 3 are reliable. In Fig. 7 we show the evolution of the fluxes at the observed epochs. To compare with the X-ray and radio flux evolution, the fluxes in the figure have been normalized to the October 2002 level, i.e., epoch 3. From this figure it is seen that the [Fe X], [Fe XI] and [Fe XIV] lines evolve very similar to the flux evolution of the soft X-rays, and considerably faster than the hard X-rays or the radio. The [Ne V], [Ar V], and [Fe VII] lines, however, increase considerably more slowly. We return to this result in Sect. 5.

Although there seem to be considerable differences between the line profiles of the different ions, it is important to realize that both the deblending procedure and the low $\mathrm{S} / \mathrm{N}$ for the faintest lines may introduce considerable uncertainties. Only for lines of high $\mathrm{S} / \mathrm{N}$ is it meaningful to compare the line profiles directly. The velocities of the different lines will be discussed in detail in Gröningsson et al. (2006). Here we only make some brief remarks about the most relevant issues. If we compare the
Table 2. Fluxes (uncorrected for reddening) of the high ionization lines from the north side of the shocked ring.

\begin{tabular}{|c|c|c|c|c|}
\hline$\overline{\text { Ion }}$ & $\begin{array}{c}\text { Rest wavel. } \\
\AA\end{array}$ & Epoch & $\begin{array}{c}\text { Flux } \\
10^{-16} \mathrm{erg} \mathrm{s}^{-1} \mathrm{~cm}^{-2}\end{array}$ & $\begin{array}{l}\text { Extinction } \\
\text { correction }\end{array}$ \\
\hline \multirow[t]{4}{*}[\mathrm{Ne}\mathrm{V}]{} & 3425.86 & 2 & - & 2.06 \\
\hline & & 3 & $5.25 \pm 0.61$ & \\
\hline & & 4 & $8.58 \pm 1.26$ & \\
\hline & & 5 & $9.69 \pm 1.83$ & \\
\hline \multirow[t]{4}{*}{ [Ar V] } & 7005.67 & 2 & - & 1.41 \\
\hline & & 3 & $0.34 \pm 0.07$ & \\
\hline & & 4 & $0.31 \pm 0.09$ & \\
\hline & & 5 & $0.35 \pm 0.08$ & \\
\hline \multirow[t]{4}{*}{ [Fe VII] } & 6087.0 & 2 & - & 1.50 \\
\hline & & 3 & $0.80 \pm 0.11$ & \\
\hline & & 4 & $1.23 \pm 0.15$ & \\
\hline & & 5 & $1.50 \pm 0.26$ & \\
\hline \multirow[t]{4}{*}[\mathrm{Fe}\mathrm{X}]{} & 6374.51 & 2 & $0.51 \pm 0.11$ & 1.47 \\
\hline & & 3 & $3.11 \pm 0.24$ & \\
\hline & & 4 & $6.54 \pm 0.37$ & \\
\hline & & 5 & $9.27 \pm 0.69$ & \\
\hline \multirow[t]{4}{*}[\mathrm{Fe}\mathrm{XI}]{} & 7891.94 & 2 & $0.91 \pm 0.17$ & 1.32 \\
\hline & & 3 & $2.98 \pm 0.25$ & \\
\hline & & 4 & $9.10 \pm 0.40$ & \\
\hline & & 5 & $9.65 \pm 0.34$ & \\
\hline \multirow[t]{4}{*}{ [Fe XIV] } & 5302.86 & 2 & $0.67 \pm 0.12$ & 1.61 \\
\hline & & 3 & $3.89 \pm 0.31$ & \\
\hline & & 4 & $11.00 \pm 0.39$ & \\
\hline & & 5 & $14.89 \pm 0.69$ & \\
\hline
\end{tabular}

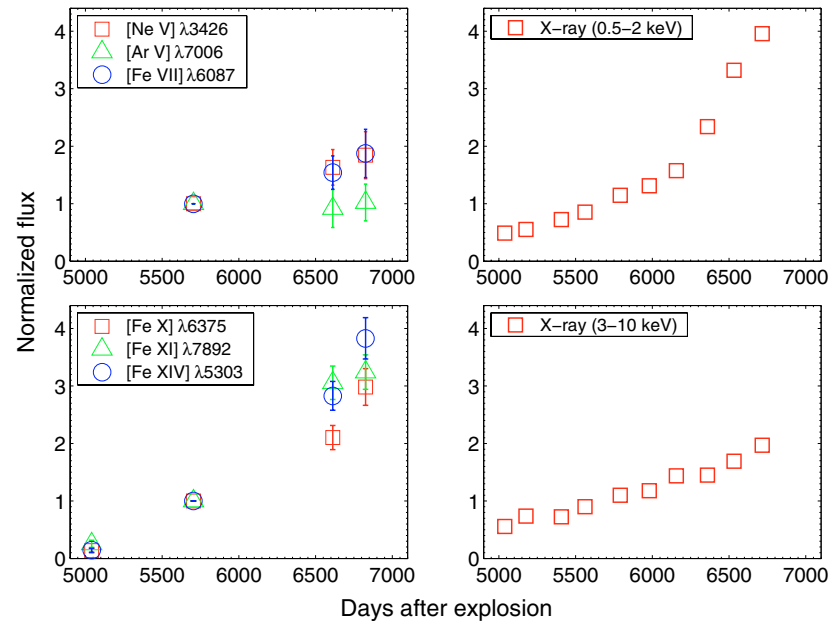

Fig. 7. Evolution of fluxes of the high ionization lines compared to that of the X-ray flux from Park et al. (2005a).

red extension of [Fe XIV] $\lambda 5302.9$ with the $[\mathrm{Fe}$ II] $\lambda 7155.2$ line we find evidence for an extension to $\sim 350 \mathrm{~km} \mathrm{~s}^{-1}$ for [Fe XIV], while the [Fe II] line extends to $\sim 250 \mathrm{~km} \mathrm{~s}^{-1}$. There are, however, important systematics due to uncertainties in background subtraction both in the spatial and dispersion directions. In addition, deblending of line profiles gives rise to uncertainties especially when the deblended line is strong compared to the line in consideration. This makes e.g., the extent of the blue wing of the [Fe XIV] 15302.9 line more uncertain than the red. With the systematic uncertainties included we estimate that the values for maximum velocities should be accurate to within $50 \mathrm{~km} \mathrm{~s}^{-1}$. 


\section{Origin of the coronal emission}

The intermediate component of both the [O III] $\lambda 5006.8$ and the [Ne V] $\lambda 3425.9$, as well as several strong UV lines, like N V $\lambda \lambda 1238.8,1242.8$, Si IV $\lambda \lambda 1393.8,1403.8$, C IV $\lambda \lambda 1548.2$, 1550.8, C II $\lambda 2325$ and Mg II $\lambda \lambda 2795.5,2802.7$, were seen in the HST spectra of Pun et al. (2002). However, no coronal lines were seen probably due to the lower spectral resolution, as well as the lower $\mathrm{S} / \mathrm{N}$. The [Fe X] $\lambda 6374.5$ and [Fe XIV] $\lambda 5302.9$ coronal lines have, however, been observed in several supernova remnants, like the Cygnus loop (Woodgate et al. 1974), Pup A, RCW86 (Lucke et al. 1979) and IC433 (Woodgate et al. 1979).

Pun et al. (2002) propose that the intermediate component arises when the blast wave hits dense protrusions from the ring. The velocity of the shocked gas behind the transmitted shock is given by $\sim\left(\rho_{\mathrm{HII}} / \rho_{\text {spot }}\right)^{1 / 2} V_{\text {blast }}$. Here $\rho_{\mathrm{HII}}$ is the density of the $\mathrm{H}$ II region of the progenitor star. Estimates give $\rho_{\mathrm{HII}} \sim 10^{2} \mathrm{~cm}^{-3}$ (Chevalier \& Dwarkadas 1995), while the density of the hot spot should be close to that of the main ring material $\sim 10^{4} \mathrm{~cm}^{-3}$. For a blast wave velocity of $\sim 3800 \mathrm{~km} \mathrm{~s}^{-1}$ (Park et al. 2005b) the velocity of the transmitted shock should be $\sim 0.1 V_{\text {blast }} \approx$ $300-500 \mathrm{~km} \mathrm{~s}^{-1}$. This is close to that observed from the intermediate component.

The origin of the X-ray emission is, however, not so clear. Part of the X-rays may arise from the blast wave propagating with a velocity of $\sim 3500 \mathrm{~km} \mathrm{~s}^{-1}$ into the H II region of the progenitor, interior to the equatorial ring. Zhekov et al. (2005), however, find a velocity from the line profiles much smaller than that inferred from the expansion from the size of the X-ray image (Park et al. 2004) and the radio source (Manchester et al. 2002, 2005), which implies an expansion rate of $\sim 3000 \mathrm{~km} \mathrm{~s}^{-1}$.

Zhekov et al. therefore propose a scenario where the blast wave propagates with this comparatively high velocity inside the ring in a medium which is dominated by a density characteristic of the $\mathrm{H}$ II region, $\sim 10^{2} \mathrm{~cm}^{-3}$. In this scenario most of the X-ray line emission arises where the blast wave hits the dense protrusions from the ring, forming transmitted shocks into the dense gas. In addition to this, Borkowski et al. (1997) find that there will be reflected shocks going back from the hot spot into the shocked gas behind the blast wave. The velocity of the shocked gas behind the reflected shock is $\sim\left(\rho_{\text {HII }} / \rho_{\text {blast }}\right)^{1 / 2} V_{\text {blast }} \approx$ $0.5 V_{\text {blast }} \approx 1000-1500 \mathrm{~km} \mathrm{~s}^{-1}$. This, and the even lower velocity from the transmitted shocks, explains the low velocity compared to the blast wave inferred from the X-ray line emitting gas. The higher density behind reflected shocks, as well as the transmitted, causes these to dominate the X-ray emission over the blast wave. It also suggests that most of the X-ray emission should be correlated with the optical emission from the hot spots. Because of the lower density and higher temperature behind the reflected shocks, these are likely to be adiabatic. The cooling gas seen in the coronal lines, as well as in the optical/UV emission from lower ionization stages, is therefore likely to arise in the radiative shocks in the hot spots. We will therefore investigate this possibility more quantitatively below.

For a gas with $x \equiv n(\mathrm{He}) / n(\mathrm{H})=0.25$ (Lundqvist \& Fransson 1996) the temperature behind the shock going into the hot spot is

$$
\begin{aligned}
T_{\mathrm{s}} & =2.27 \times 10^{-9} \frac{(1+4 x)}{(2+3 x)} V_{\mathrm{s}}^{2} \\
& =1.5 \times 10^{6}\left(\frac{V_{\mathrm{s}}}{300 \mathrm{~km} \mathrm{~s}^{-1}}\right)^{2} \mathrm{~K} .
\end{aligned}
$$

Michael et al. (2002) find that for a shock with velocity $500 \mathrm{~km} \mathrm{~s}^{-1}$ equipartition between electrons and ions takes place on a time scale of $\sim 60\left(n_{\mathrm{e}} / 10^{4} \mathrm{~cm}^{-3}\right)^{-1}$ days. Shocks with lower velocity will equilibrate faster. In the following we therefore assume that $T_{\mathrm{e}}=T_{\mathrm{ion}}=T_{\mathrm{s}}$.

For $100 \lesssim V_{\mathrm{s}} \lesssim 600 \mathrm{~km} \mathrm{~s}^{-1}$ the cooling function for the CNO-enriched composition found for the ring in Lundqvist \& Fransson (1996) is $\Lambda\left(T_{\mathrm{e}}\right) \approx 4.0 \times$ $10^{-23}\left(T_{\mathrm{e}} / 10^{6} \mathrm{~K}\right)^{-0.7} \mathrm{erg} \mathrm{s}^{-1} \mathrm{~cm}^{3}$, using the code by Nymark et al. (2005). The cooling time of the shock may therefore be approximated by

$t_{\text {cool }} \approx 8.3\left(\frac{n_{\text {spot }}}{10^{4} \mathrm{~cm}^{-3}}\right)^{-1}\left(\frac{V_{\mathrm{s}}}{300 \mathrm{~km} \mathrm{~s}^{-1}}\right)^{3.4}$ years,

where $n_{\text {spot }}$ is the pre-shock density. Therefore, depending on the density and the shock velocity, the shock may be either adiabatic or radiative. While coronal emission lines may arise in any hot gas with a temperature of $\sim 10^{6} \mathrm{~K}$, the wide range of ionization stages with similar line profiles seen in the intermediate velocity component, from Fe II to Fe XIV, strongly indicates that at least some of the emission is originating in radiative shocks.

The structure of the radiative shocks was discussed in Pun et al. (2002). Pun et al., however, mainly focused on low and intermediate ionization lines. To calculate the flux of the opti$\mathrm{cal} / \mathrm{UV} / \mathrm{IR}$ coronal lines, as well as some of the other high ionization lines, we compute the spectrum of the shock, using the shock code of Nymark et al. (2005). Although originally used for the reverse shock in supernovae, this is, of course, applicable for any radiative shock, independent of shock direction. This determines the temperature and ionization structure of the shocked gas by solving the time dependent ionization and hydrodynamic equations, assuming that the structure is stationary and one-dimensional. The latter should be sufficient as long as the shock is radiative. Collisional ionization rates, as well as recombination rates, are from the most recent data, and references are given in Nymark et al. (2005). In addition, we include charge transfer, which becomes important at these comparatively low temperatures. The uncertainties of the ionization and recombination data especially for iron have been discussed by Masai (1997) and Gianetti et al. (2000). The emissivity is calculated using full multi-level atoms. Pre-ionization of the unshocked gas by X-rays from the shock is calculated using an updated version of the code in Chevalier \& Fransson (1994). The abundances used are the ones used by Pun et al. (2002), which are the $\mathrm{CNO}$ enriched abundances for $\mathrm{He}, \mathrm{C}, \mathrm{N}$ and $\mathrm{O}$, taken from Lundqvist \& Fransson (1996), and LMC abundances from Russell \& Dopita (1992) for the other elements, with exception to Si which is from Welty et al. (1999).

Because the focus in this paper is on the coronal lines we do not attempt to calculate the structure of the photoionization heated gas behind the cooling, shocked gas. This region dominates the flux for medium and low ionization lines, like [O III], $[\mathrm{Ne} \mathrm{III}]$ and lower ionization stages. The structure of this region, as well as the pre-ionized emission lines, will be discussed in a separate paper. Here we only note that the low and intermediate ionization lines, like [O III], [Ne V], [Ar V], and [Fe VII], all have narrow components at zero velocity. The higher ionization lines, [Fe X], [Fe XI], and [Fe XIV], however, lack such a component. This indicates that pre-ionization of the unshocked gas does not reach more than the former stages. Models similar to those in Lundqvist \& Fransson (1996) show that gas ionized by the initial UV/soft X-ray flash at shock break out with densities $\gtrsim 10^{4} \mathrm{~cm}^{-3}$ is at these epochs mainly found in Fe I-II, with $\lesssim 10^{-3}$ in Fe III. Only in gas with densities $\lesssim 10^{3} \mathrm{~cm}^{-3}$ can ions like Fe VII be found. 


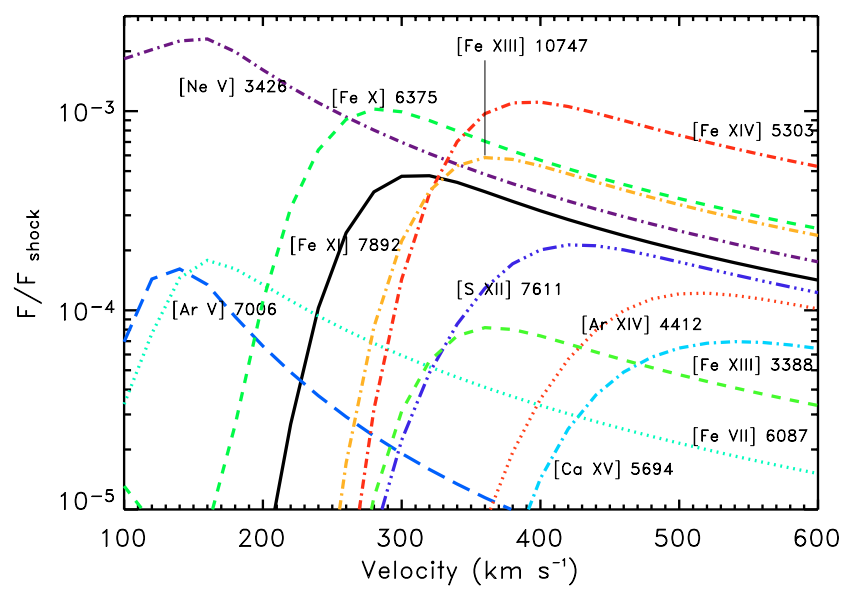

Fig. 8. Fluxes of the most important optical lines from radiative shocks as function of the shock velocity. The fluxes are given relative to the energy flux, $F_{\text {shock }}=\rho V_{\text {shock }}^{3} / 2$, through the shock.

The collision strengths of the important coronal lines are mainly from the Chianti data base (Dere et al. 1997; Landi et al. 2006), but in some cases supplemented with more recent calculations. The critical densities for collisional de-excitation of the [Fe X-XIV] lines are in the range $10^{7}-10^{9} \mathrm{~cm}^{-3}$, and collisional destruction is therefore not important for these. Consequently, the relative line ratios are not sensitive to the exact value of the density. Here we take a value of $n_{\text {spot }}=10^{4} \mathrm{~cm}^{-3}$ for the preshock density.

In Fig. 8 we show the strengths of the most important optical, high ionization lines as a function of the shock velocity relative to the kinetic flux through the shock, $\rho V_{\mathrm{s}}^{3} / 2$. From this we see that at shock velocities $\$ 400 \mathrm{~km} \mathrm{~s}^{-1}$ the line ratios vary strongly with velocity, while above this velocity they are, with the exceptions of [S XII] $\lambda 7611.0$ and [Ar XIV] $\lambda 4412.2$, nearly constant. This is explained by the fact that above $\sim 400 \mathrm{~km} \mathrm{~s}^{-1}$ the most abundant ions in the immediate post-shock gas are ions more highly ionized than $\mathrm{Ne} \mathrm{V}$ and Fe XIV. For a radiative shock with velocity higher than $\sim 400 \mathrm{~km} \mathrm{~s}^{-1}$ the fraction of the cooling region above $\sim 2 \times 10^{6} \mathrm{~K}$, which corresponds to ionization stages higher than these ions, is largely irrelevant to the flux of the coronal lines, and the line ratios will therefore be insensitive to the shock velocity.

In Fig. 9 we show the ionization and temperature structure of iron of a radiative shock with velocity $350 \mathrm{~km} \mathrm{~s}^{-1}$. Immediately behind the shock in a region too small to be well resolved on this scale, $\sim 5 \times 10^{13} \mathrm{~cm}$, the ions adjust from their pre-ionization values to their equilibrium abundances. Behind this region Fe IX-XIV all have high abundances in most of the shocked gas. As the gas cools the temperature and ionization slowly decreases. When the temperature has fallen below $\sim 10^{6} \mathrm{~K}$ cooling increases catastrophically and drops to $(1-3) \times$ $10^{4} \mathrm{~K}$. This region is too thin to be resolved in the plot, but is very important for the observed emission lines (see below). The different lines are therefore direct probes of the temperature interval where they have their maximum abundance.

To more clearly illustrate the sensitivity of the different lines to the temperature we show in Fig. 10 the contribution to the luminosity per logarithmic temperature interval of each line as function of temperature. This clearly shows how the different lines trace different temperature ranges behind the shock, with the higher ionization stages naturally more sensitive to the higher temperatures. Consequently, the [Fe XI-XIV] lines arise

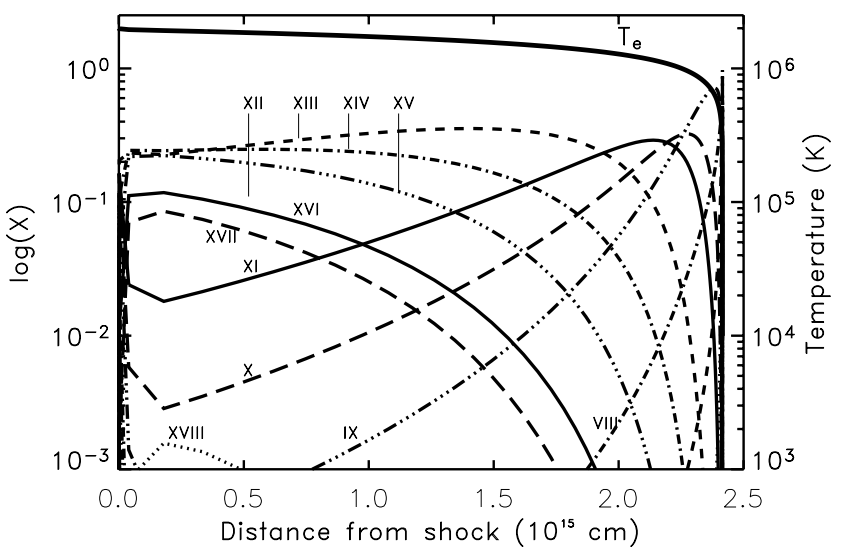

Fig. 9. Ionization structure for iron and temperature behind a radiative shock with velocity $350 \mathrm{~km} \mathrm{~s}^{-1}$ and pre-shock density $10^{4} \mathrm{~cm}^{-3}$.

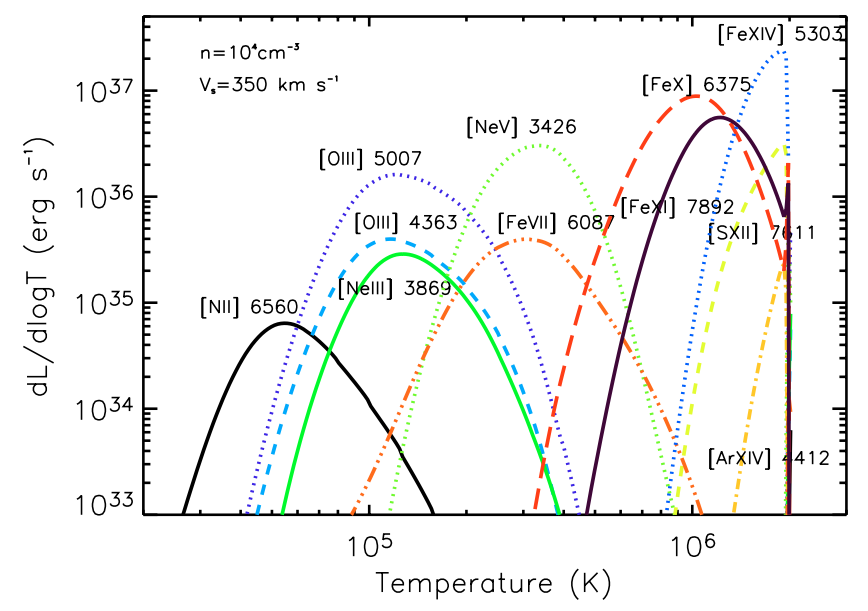

Fig. 10. Contribution to the luminosity per logarithmic temperature interval to different lines behind a shock with velocity $350 \mathrm{~km} \mathrm{~s}^{-1}$.

at $(1-2) \times 10^{6} \mathrm{~K}$, while the $[\mathrm{Ne} \mathrm{V}],[\mathrm{Ar} \mathrm{V}]$ and [Fe VII] lines arise at $\sim 3 \times 10^{5} \mathrm{~K}$ in a geometrically narrow, but important, region, where the temperature falls from $10^{6} \mathrm{~K}$ to $10^{4} \mathrm{~K}$. Because the whole post-shock region is in near pressure equilibrium, the density is $n_{\mathrm{e}}\left(T_{\mathrm{e}}\right) \approx 4 n_{\mathrm{spot}} T_{\mathrm{s}} / T_{\mathrm{e}}$. The [Ne V] and [Fe VII] lines e.g., arise at $T_{\mathrm{e}} \sim 3 \times 10^{5} \mathrm{~K}$, corresponding to $\sim 24 n_{\text {spot }} \approx$ $2.4 \times 10^{5} \mathrm{~cm}^{-3}$, for a pre-shock density of $n_{\text {spot }}=10^{4} \mathrm{~cm}^{-3}$. The critical densities for these lines are $\gtrsim 10^{7} \mathrm{~cm}^{-3}$, and collisional de-excitation is therefore clearly not important for these.

For completeness Fig. 10 also shows the [N II], [O III] and [Ne III] lines. In the collisionally ionized part of the shock they each trace different temperature intervals from $\sim 10^{4} \mathrm{~K}$ to $\sim 4 \times 10^{5} \mathrm{~K}$. They all have, however, more important contributions from the photoionized, cooling gas, not included in these models.

To compare the models with our observations we relate all line fluxes to that of [Fe XIV] $\lambda 5302.9$ at the different epochs. These ratios are given for the different epochs in Table 3. In the same Table we also give the shock velocity corresponding to the different line ratios, taken from Fig. 8. As can be seen, the range of shock velocities is for all line ratios surprisingly small, 310-390 $\mathrm{km} \mathrm{s}^{-1}$. The corresponding temperature behind the shock is $(1.6-2.5) \times 10^{6} \mathrm{~K}$. This comparison assumes that the emitting area in each line is the same. If there are shocks with a range of velocities this assumption may be questionable. Because of the rapid variation of the line ratios with velocity, this 
Table 3. Reddening corrected fluxes relative to [Fe XIV] $\lambda 5303$ and corresponding shock velocities $\left(\mathrm{km} \mathrm{s}^{-1}\right)$.

\begin{tabular}{lrrrr}
\hline \hline Line & Epoch 2 & Epoch 3 & Epoch 4 & Epoch 5 \\
\hline$[\mathrm{Fe} \mathrm{VII}] \lambda 6087.0$ & - & 0.19 & 0.10 & 0.094 \\
& - & 316 & 330 & 332 \\
{$[\mathrm{Fe}$ X] $]$ 6374.5 } & 0.65 & 0.68 & 0.54 & 0.57 \\
& 369 & 366 & 391 & 381 \\
{$[\mathrm{Fe}$ XI] $] \lambda 7892.0$} & 1.05 & 0.63 & 0.68 & 0.53 \\
& 326 & 340 & 338 & 348 \\
{$[\mathrm{Ne}$ V] $] \lambda 3425.9$} & - & 1.74 & 1.00 & 0.83 \\
& - & 310 & 318 & 321 \\
{$[\mathrm{Ar} \mathrm{V}] \lambda 7005.7$} & - & 0.076 & 0.025 & 0.021 \\
& - & 313 & 336 & 339 \\
\hline
\end{tabular}

is, however, not likely to affect the derived velocities by a large factor.

There is some tendency for lines from ions with lower ionization potential, e.g., $\mathrm{Ne} \mathrm{V}, \mathrm{Ar} \mathrm{V}$ and Fe VII, to give a somewhat lower velocity than the lines from high ionization ions like Fe X and Fe XI. The fact that we do not see [Ar XIV] 14412.2, at a flux level of $\$ 15 \%$ of the [Fe XIV] line is consistent with a shock velocity $\lesssim 500 \mathrm{~km} \mathrm{~s}^{-1}$. The [Ca XV] $\lambda 5694.4$ line gives a similar upper limit of $\sim 500 \mathrm{~km} \mathrm{~s}^{-1}$ (Fig. 8).

Finally, we note that although only barely covered by these observations, one also expects the [Fe XIII] $\lambda 3388.5$ and $\lambda \lambda 10746.8-10797.9$ lines to be detectable in the UV and near-IR, respectively. In particular, the IR lines are expected to have a strength comparable to that of $[\mathrm{Fe} \mathrm{X}] \lambda 6374.5$ (see Fig. 8). The $\lambda 3388.5$ line is within our observed range, but the spectrum is here noisy due to the decreasing sensitivity of the instrument and the influence of the atmosphere. We estimate a maximum strength comparable to that of [Fe XIV] 15302.9. Because the expected flux is $\sim 10 \%$ of this line, this limit is not very useful. The IR lines are outside the range.

\section{Discussion}

Although we get good agreement for a rather narrow range of shock velocities, it is clear that the dynamics is more complex. This can be seen in the hydrodynamic simulations of the collision performed by several groups (Luo et al. 1994; Masai \& Nomoto 1994; Borkowski et al. 1997). In particular, Borkowski et al. (1997) have modeled the structure and X-ray emission from the impact with 2D hydro simulations. The interplay of the shock propagating into the dense ring gas and reflected shocks from the ejecta-CSM gives a time dependence, as well as spatial dependence, which is complex and only can be described by detailed multi-dimensional hydrodynamical simulations. Although the Borkowski et al. simulations used a simplified model for the ring structure, they give a good idea of the processes and complications involved in modelling the emission.

A further complication is that the observed spectra are a convolution of several hot spots seen with different projected velocities. In addition, the number of spots is increasing. In these respects our observations have similar limitations as the Chandra $\mathrm{X}$-rays observations, although our spectral resolution is much better. Spatially resolved spectroscopy would be of obvious importance.

In Sect. 3 we found that the [Fe XIV] $\lambda 5302.9$ line extends to $\sim 350 \mathrm{~km} \mathrm{~s}^{-1}$, while the lower ionization lines only extend to $\sim 250 \mathrm{~km} \mathrm{~s}^{-1}$. This may indicate that the low ionization lines have a dominant contribution from tangential shocks with lower velocity, while the high ionization lines also come from shocks perpendicular to the blast wave, having a high velocity.

Michael et al. (2002) find that the relative fluxes of the lines observed with the grating spectrometer on Chandra in October 1999 are best fitted with a shock temperature of $k T_{\mathrm{e}} \sim$ $2.9 \pm 0.4 \mathrm{keV}$. The lower dispersion CCD spectra obtained in December 2000 indicated a marginally lower temperature, $k T_{\mathrm{e}} \sim 2.7 \pm 0.2 \mathrm{keV}$. In addition, they find that they get a slightly better fit to the spectrum with an additional softer component with $k T_{\mathrm{e}} \sim 0.4 \pm 0.1 \mathrm{keV}$. The flux contribution from this was $\sim 7 \%$. It is worth noting that even the high temperature component has an electron temperature that is considerably lower than the shock temperature, corresponding to equipartition, $\sim 17 \mathrm{keV}$. This is in common with several other SNRs.

Due to the increase in the X-ray flux Zhekov et al. (2005, 2006) were able to repeat this study with considerably higher $\mathrm{S} / \mathrm{N}$. Zhekov et al. find that they get a good fit to the X-ray line ratios, as well as the widths, for a model with a range of temperature, $0.15-4 \mathrm{keV}$, corresponding to $V_{\mathrm{s}}=340-1700 \mathrm{~km} \mathrm{~s}^{-1}$. The lower part of this range is similar to that observed in the optical lines, and it is very likely that they arise from the same region. As Zhekov et al. (2005) remark, the upper range of velocities is, however, likely to come from adiabatic shocks, probably reflected from the impact on the protrusions. If they are adiabatic and with velocity $\gtrsim 400 \mathrm{~km} \mathrm{~s}^{-1}$, corresponding to a temperature of $\gtrsim 3 \times 10^{6} \mathrm{~K}$, they will not produce optical emission.

We propose that the regions that produce the soft X-ray flux also account for most of the emission in the $[\mathrm{Fe} X],[\mathrm{Fe} \mathrm{XI}]$ and [Fe XIV] lines. This is supported by the similar evolution of the flux between these two components (Fig. 7), and, as mentioned above, the comparable shock velocities required to produce them, $\sim 350 \mathrm{~km} \mathrm{~s}^{-1}$ (see also Table 3 ). However, in order to explain the slower evolution of the intermediate ionization lines such as [Ne V], [Ar V] and [Fe VII], we do require that some fraction of the [Fe X-XIV] fluxes arise from adiabatic shocks which would not be contributing to the intermediate and low ionization lines. The sensitivity of the cooling time scales to the shock velocities and the density of the hot spots (Eq. (2)) makes this a plausible scenario. The somewhat narrower line widths that the intermediate ionization lines exhibit also support this argumentation.

\section{Conclusions and summary}

The most important result of this paper is the discovery of a number of high ionization lines coming from gas of temperatures up to $\sim 2 \times 10^{6} \mathrm{~K}$. The rapidly increasing flux of these is well correlated to the flux in the soft X-rays, and offer a complimentary view of the interaction of the ejecta and the ring material. The large range in ionization stage with similar line profiles shows that most of the emission is coming from radiative shocks with velocity $310-390 \mathrm{~km} \mathrm{~s}^{-1}$, although a fraction of the coronal line emission may originate in adiabatic shocks. The shock velocity we find from our spectral modeling is consistent with the width of the lines. We hope in the future to do a more detailed modeling of the spectra, including both the lower ionization lines from the photoionized, cooling gas, as well as the X-ray lines. Also a more detailed modeling of the hydrodynamics of the explosion along the lines of Borkowski et al. (1997) with these extra constraints from the line strengths, as well as the line profiles would be highly interesting. Continued monitoring of the ring collision is of obvious high importance. The increasing flux, as well as new instruments using adaptive optics, will enable us to study this unique collision in even more detail. 
Acknowledgements. We are grateful to the referee for several useful comments. This work was supported in part by the Swedish Research Council and the Swedish National Space Board. Part of this research was performed while CF and RAC were visiting the Kavli Institute of Theoretical Physics, supported in part by the National Science Foundation under Grant No. PHY99-07949. PL is a Research Fellow at the Royal Swedish Academy supported by a grant from the Wallenberg Foundation.

\section{References}

Borkowski, K. J., Blondin, J. M., \& McCray, R. 1997, ApJ, 477, 281

Bouchet, P., et al. 2006 [arXiv: astro-ph/0601495]

Cardelli, J. A., Clayton, G. C., \& Mathis, J. S. 1989, ApJ, 345, 245

Chevalier, R. A., \& Dwarkadas, V. V. 1995, ApJ, 452, L45

Chevalier, R. A., \& Fransson, C. 1994, ApJ, 420, 268

Dekker, H., D’Odorico, S., Kaufer, A., Delabre, B., \& Kotzlowski, H. 2000, Proc. SPIE, 4008, 534

Dere, K. P., Landi, E., Mason, H. E., Monsignori Fossi, B. C., \& Young, P. R. 1997, A\&AS, 125, 149

Fitzpatrick, E. L. 1999, PASP, 111, 63

Fitzpatrick, E. L., \& Walborn, N. R. 1990, AJ, 99, 1483

Gaensler, B. M., Manchester, R. N., Staveley-Smith, L., et al. 1997, ApJ, 479, 845

Gianetti, D., Landi, E., \& Landini, M. 2000, A\&A, 360, 1148

Gröningsson, P., et al. 2006, in preparation

Landi, E., Del Zanna, G., Young, P. R., et al. 2006, ApJS, 162, 261

Lucke, R. L., Woodgate, B. E., Culhane, J. L., Socker, D. G., \& Zarnecki, J. C. 1979, ApJ, 228, 763

Luo, D., McCray, R., \& Slavin, J. 1994, ApJ, 430, 264
Lundqvist, P., \& Fransson, C. 1996, ApJ, 464, 924

Manchester, R. N., Gaensler, B. M., Wheaton, V. C., et al. 2002, PASAu, 19, 207 Manchester, R. N., Gaensler, B. M., Staveley-Smith, L., Kesteven, M. J., \& Tzioumis, A. K. 2005, ApJ, 628, L131

Masai, K. 1997, A\&A, 324, 410

Masai, K., \& Nomoto, K. 1994, ApJ, 424, 924

McCray, R. 2005, in Cosmic Explosions, On the 10th Anniversary of SN1993J, ed. J. M. Marcaide, \& Kurt W. Weiler (Springer), IAU Colloq., 192, 77

Michael, E., McCray, R., Pun, C. S. J., et al. 1998, ApJ, 509, L117

Michael, E., McCray, R., Pun, C. S. J., et al. 2000, ApJ, 542, L53

Michael, E., Zhekov, S., McCray, R., et al. 2002, ApJ, 574, 166

Nymark, T. K., Fransson, C., \& Kozma, C. 2006, A\&A, 449, 171

Park, S., Zhekov, S. A., Burrows, D. N., Garmire, G. P., \& McCray, R. 2004, ApJ, 610, 275

Park, S., Zhekov, S. A., Burrows, D. N., \& McCray, R. 2005a, ApJ, 634, L73

Park, S., Zhekov, S. A., Burrows, D. N., et al. 2005b

[arXiv:astro-ph/0511355]

Pun, C. S. J., Michael, E., Zhekov, S. A., et al. 2002, ApJ, 572, 906

Russell, S. C., \& Dopita, M. A. 1992, ApJ, 384, 508

Smith, N., Zhekov, S. A., Heng, K., et al. 2005, ApJ, 635, L41

Staveley-Smith, L., Kim, S., Calabretta, M. R., Haynes, R. F., \& Kesteven, M. J. 2003, MNRAS, 339, 87

Welty, D. E., Frisch, P. C., Sonneborn, G., \& York, D. G. 1999, ApJ, 512, 636

Woodgate, B. E., Stockman, H. S., Angel, J. R. P., \& Kirshner, R. P. 1974, ApJ, 188, L79

Woodgate, B. E., Lucke, R. L., \& Socker, D. G. 1979, ApJ, 229, L119

Zhekov, S. A., McCray, R., Borkowski, K. J., Burrows, D. N., \& Park, S. 2005, ApJ, 628, L127

Zhekov, S. A., McCray, R., Borkowski, K. J., Burrows, D. N., \& Park, S. 2006 [arXiv:astro-ph/0603305] 